\title{
Comparative analysis of male androgen responsiveness to social environment in birds: the effects of mating system and paternal incubation
}

\author{
Katharina Hirschenhauser, ${ }^{\mathrm{a}, *}$ Hans Winkler, ${ }^{\mathrm{b}}$ and Rui F. Oliveira ${ }^{\mathrm{a}}$ \\ ${ }^{a}$ ISPA, Unidade de Investigação em Eco-Etologia, Rua Jardim do Tabaco 34, P-1149-041 Lisbon, Portugal \\ ${ }^{\mathrm{b}}$ KLIVV, Akademie der Wissenschaften, Savoyenstrasse 1a, A-1160 Vienna, Austria
}

Received 1 September 2001; revised 13 January 2003; accepted 14 January 2003

\begin{abstract}
Male androgen responses to social challenges have been predicted to vary with mating system, male-male aggressiveness, and the degree of paternal investment in birds ("challenge hypothesis," Am. Nat. 136 (1990), 829). This study focused on the interspecific predictions of the challenge hypothesis. Comparative methods were used to control for effects of the phylogenetic relatedness among the sampled species. Male androgen data of 84 bird species were collected from literature records on seasonal androgen patterns. From these, the androgen responsiveness (AR) was calculated as described in the original challenge hypothesis (i.e., maximum physiological level/breeding baseline). Scatterplots of AR versus mating strategy, male-male aggressiveness, and the degree of paternal care confirmed the expected interspecific patterns. When phylogenetic analyses were performed among all of the sampled species, the effects of paternal investment disappeared while the AR remained covarying to a high degree with mating system and male-male aggressiveness. Although these mechanisms may be different at the intraspecific level, this suggests that interspecific differences of AR in male birds may have evolved in response to changes of mating strategies, rather than in response to altered paternal duties. However, control for phylogeny among the subsample of 32 passerine species revealed that if any paternal investment contributed to the observed variance in AR, then the change from "no male incubation" to "male shares incubation duties" represented the most effective, whereas the male's contribution to feeding offspring did not explain the observed variation of AR.
\end{abstract}

(C) 2003 Elsevier Science (USA). All rights reserved.

Keywords: Birds; Challenge hypothesis; Testosterone; Androgen responsiveness; Mating system; Paternal care; Incubation; Male-male aggression; Comparative analysis

To optimize their reproductive output, males have to trade-off between mating, which requires high levels of androgens, and parenting, which requires low androgen levels (Hegner and Wingfield, 1987). Variation of androgen levels above a reproductive baseline (i.e., the minimum amount needed for the expression of secondary sex characters, the maturation of the gonads, and the expression of reproductive behavior) within wild populations of birds may be explained by the flexibility of the individual androgen responsiveness (AR) to the social environment (Wingfield et al., 1990). In particular, it was proposed that males'

* Corresponding author.

E-mail address: katarina@ispa.pt (K. Hirschenhauser). androgen levels should be responsive to social challenges imposed by other males in competition for mates and territories ("challenge hypothesis"; Wingfield et al., 1990). In monogamous species with paternal care, male-male competition for mates should be lower than in polygynous species without paternal care. Therefore, one of the predictions of the challenge hypothesis is that the AR to social challenges should vary with mating strategy and the degree of paternal investment (Wingfield et al., 1990, 2000). Monogamous male birds with a high degree of paternal care are predicted to have low breeding baseline androgen levels and will remain highly responsive to social challenges (e.g., territorial intrusions) throughout the breeding season. In contrast, males with a high degree of polygyny and low or 
no paternal care are predicted to have high androgen levels throughout all breeding phases and thus, to exhibit only small further increases in response to social challenges (Beletsky et al., 1989). These predictions have been confirmed by a number of correlational studies in free-living bird populations (Rissman and Wingfield, 1984; Wingfield, 1984a; Wada et al., 1999; Wikelski et al., 1999; Hirschenhauser et al., 2000; Wingfield et al., 2000). Moreover, exogenous administration of testosterone was shown to inhibit the transition from mating to paternal behavior, and to promote polygynous mating strategies in monogamous biparental populations of various passerine species (Silverin, 1980; Wingfield, 1984b; Hegner and Wingfield, 1987; Dittami et al., 1991; Ketterson and Nolan, 1992; De Ridder et al., 2000). However, a hormonal signal may not only interact with behavior, physiology, and morphology, but in addition, it may involve potential "costs" in terms of overall fitness, such as direct and indirect energetic costs, immunosuppression, increased mortality, and reproduction failure (Marler and Moore, 1988; Dufty, 1989; Wingfield, 1990; Ros et al., 1997; Wingfield et al., 1999a; Peters, 2000). As these changes in fitness occur where the same hormone mediates antagonistic traits, androgens can be viewed as physiological mediators of the trade-off between investment in male-male competition or in paternal care (Hegner and Wingfield, 1987; Ketterson and Nolan, 1992). Therefore, hormonal mechanisms, like other phenotypic traits, may be targets of selection (Ketterson and Nolan, 1992; Houck and Woodley, 1995). At this stage it is important to point out the importance of distinguishing between the intraspecific and the interspecific level of the predicted interactions because correlations among traits within a species may not necessarily correspond to correlations among species (Ptacek and Travis, 1998). For instance, among the cooperatively breeding Acrocephalus warblers helping species occur in poor habitats, while within species helpers are more numerous at good sites (Leisler et al., 2002). Accordingly, the predicted interactions between mating strategy, paternal care, and AR to social challenges at the intraspecific level do not necessarily have to act equally at the interspecific level. Here, the predictions of the challenge hypothesis were tested at the interspecific level. We analyzed the effects of mating strategy and degree of paternal care on AR to social challenges in males from different bird species and added a control for the phylogenetic relatedness among the sampled species.

\section{Methods}

We carried out a literature survey collecting measures of the potential AR to social challenges of males from different bird species in order to reproduce and to expand the size of the original sample (Wingfield et al., 1990: 20 species; Wingfield et al., 2000: 60 species). The references given in the original challenge hypothesis were scanned as well as the articles found in online database surveys (Cambridge
Table 1

Distribution of the different categories of mating system and paternal care among the 84 sampled species

\begin{tabular}{lr}
\hline Mating system & \\
\hline Monogamous & $72.6 \%$ \\
Polyandrous & $3.6 \%$ \\
Polygynous & $20.2 \%$ \\
Promiscuous & $3.6 \%$ \\
Parenting system & \\
Nonpaternal & $16.7 \%$ \\
Biparental & $76.2 \%$ \\
Paternal & $7.1 \%$ \\
\hline
\end{tabular}

University Abstracts; IDEAL-Library; NISC-Biblioline; PubMed) that reported territorial intruder experiments (situational) or seasonal androgen patterns. From each paper the following measures were taken: (1) the "nonbreeding baseline" androgen level (level A sensu Wingfield et al., 1990: photorefractory levels or, if not given, the lower limit of reliable measurements of the immunoassays); (2) the "breeding baseline" level (level B sensu Wingfield 1990 et al.: the average androgen level throughout the different breeding phases, including parenting); and (3) the "physiological maximum" (level C sensu Wingfield, 1990 et al.: specific responses to territorial challenges, if available, or the highest androgen level observed during peak mating phases, where high frequencies of territorial male-male interactions may be assumed). Based on these measures, the potential AR for each species was expressed as the relative ratio (C-A)/(B-A) as proposed by Wingfield et al. (1990). For example, a large AR indicates that males in this species respond to territorial intruders with large androgen increases from low breeding baseline levels to the physiological maximum, whereas males from species with smaller AR have already higher breeding baseline androgen levels and, therefore, further increases in response to social stimuli are smaller (Oliveira et al., 2001a). As a rule, when more than one article was available for a given species, the one cited by Wingfield et al. $(1990,2000)$ was used, and in the case of species not present in Wingfield's database, the most recently published results were selected. When the data needed were not given in the text or in tables, the published figures were scanned and the androgen levels were extrapolated using the UnGraph software package (Biosoft, 1998).

Our database consists of 84 bird species, and is dominated by monogamous and biparental species (61 and 64 species, respectively, Table 1), which reflects the available distribution of mating and parenting systems among birds (Clutton-Brock, 1991) and echoes the distribution of social systems in the sample represented in the challenge hypothesis (Wingfield et al., 2000).

In order to reproduce the patterns from the original challenge hypothesis (Wingfield et al., 1990, 2000), information on male-male aggressiveness (competition for mates and territory), mating strategy, and the degree of 
paternal care were collected for each species as given in the original challenge hypothesis (Table 2). The degree of territorial male-male aggressiveness was rated on a three-point scale (low, moderate, high) according to the information given in the scanned article or, where not given there, as classified in Cramp (1998). Regarding mating strategy, species were assigned to one of the four categories: monogamous, polyandrous, polygynous, or promiscuous. Furthermore, the scale of the degree of paternal care (i.e., maternal, biparental, or paternal) was refined, by quantifying the males' specific contribution to the different parental phases, such as incubation and feeding offspring expressed as percentages (Møller and Cuervo, 2000; Table 2). This information was, if not given in the original articles, taken from textbooks (Morse Nice, 1943; Willis, 1972; Glutz von Blotzheim et al., 1981; Welty, 1982; Stacey and Koenig, 1990; Hoyo et al., 1992, 1996; Johnsgard, 1994; Cramp, 1998 ) or provided by the authors of the original articles. Because of the variables involving offspring feeding, these analyses included data from altricial species only. We chose to analyze the subsample of passerines ( $N=32$ species), as this is a relatively homogenous group with regard to body size, which provided sufficient variability of paternal care systems during the different breeding phases. Numbers of species may vary depending on the information available.

Because AR data were skewed, they were $\log _{10}$-transformed. First, we repeated the analysis performed in the original challenge hypothesis (i.e., Wingfield et al., 1990) by plotting the AR of the 84 species in our database by the "ratio d" (male-male aggressiveness divided by the degree of paternal care). A small ratio $d$ indicates low male-male aggressiveness and a high degree of paternal care in this species (for example Aptenodytes patagonicus; Table 2), whereas highest ratios $\mathrm{d}$ result from species with highly territorial males and no paternal care (for example Ptilonorhynchus violaceus; Table 2). Because we aimed at elaborating the specific contribution of each of the independent variables per se, we subsequently dealt with the mating strategy, the degree of paternal care, and the male-male aggressiveness categories separately.

\section{Control for phylogeny}

For the comparative analyses, we employed generalized linear methods as outlined in Dobson (1990). Models of this kind can be extended to include covariances among species due to the evolutionary process (Martins and Hansen, 1997; Rohif, 2001). Thus, the full range and power of this tool is available for statistical analyses in comparative studies, without the problems that pertain to popular algorithmic methods (e.g., independent contrasts), which produce, however, identical results under certain boundary conditions (Rohif, 2001). Multiple regression analysis was used with the independent variables coded as discrete states. In the generalized least squares (GLS) estimations, two error models were considered. The basic assumption for the analyses was that the phylogenetic variance corresponds to the Brownian motion model (Felsenstein, 1985). However, we also tested a punctuated model. Because the results did not differ between the two models and because it may not be appropriate to estimate the number of speciation events lying between two nodes from phylogenetic trees that cover widely different groups at varying resolution, we only report the results of the gradual model. The phylogeny used was derived from Sibley and Ahlquist (1990), who analyzed DNA-DNA hybridization data. Additional information came from cytochrome b studies by Ellsworth et al. (1996), Patten and Fugate (1998), Helbig and Seibold (1999), Johnson and Lanyon (1999), Kimball et al. (1999), and Klicka et al. (2000).

To control for the effect of phylogenetic relatedness, the independent variables mating system, male-male aggressiveness, and degree of paternal care were entered separately into single and multiple regression analyses (GLS) with AR as the dependent variable (Price, 1997; Garland et al., 1999; Dunn et al., 2001). Mating system was coded with three independent dummy variables (Tabachnik and Fidell, 2001 ; i.e., polyandrous $1,0,0$; monogamous $0,1,0$; polygynous $0,0,1$; and promiscuous $0,0,0$ ). Male-male aggressiveness was also coded with two dummy variables (i.e., species with low 0,0 ; moderate 1,0 ; and high territorial male-male aggressiveness 1,1), and the degree of paternal care was coded with two dummy variables that implied an order of the paternal effort (i.e., species with no paternal care 0,0 ; biparental species 1,0 ; and species with full paternal care $1,1)$. However, to rule out the potential bias due to defining the mating categories as continuous variables with equidistant phenotypes, we also repeated the analyses comparing between two discrete character states of mating system [monogamous $(N=61)$ vs polygynous $(N=17)$ ]. The alternative would have been to use ANOVA, equivalent to multiple regression on similar dummy variables, but constrained to sum up to zero (Overall and Spiegel, 1969; Dobson, 1990). Because the data are unbalanced with respect to the factors analyzed, no matter which analysis is used, the problem of colinearity and thus, deciding which variable is more influential remains. Confidence intervals of each category in the final regression models served to determine the significant contributions of each variable to the variance of the response.

\section{Social environment}

Because species living at high group densities may better cope with higher frequencies of male-male interactions, the effects of different parameters of the social environment were also included in the analyses. The mode of group living that males of different species were coping with during the breeding season was qualitatively determined [i.e., breeding density: dispersed $(N=42)$, or aggregated nesting $(N=30)$, Westneat and Sherman, 1997; Searcy et al., 1999], as well as group living outside of the breeding 
Table 2

List of all sampled species with the measured androgen responsiveness $[A R=(C-A) /(B-A)]$ and the categories classified for the phylogenetic analyses

\begin{tabular}{|c|c|c|c|c|c|c|c|c|c|c|c|c|c|}
\hline Species & AR & Mating $^{a}$ & $\begin{array}{l}\text { Parental } \\
\text { care }^{\mathrm{b}}\end{array}$ & $\begin{array}{l}\text { Territory } \\
\text { aggress. }{ }^{f}\end{array}$ & $\begin{array}{l}\text { Precocial/ } \\
\text { altricial }^{\mathrm{g}}\end{array}$ & INC\% & FO\% & $\begin{array}{l}\text { Breeding } \\
\text { density }\end{array}$ & $\begin{array}{l}\text { Group } \\
\text { living }\end{array}$ & Pairbond & $\begin{array}{l}\text { Free- } \\
\text { living/ } \\
\text { captive }\end{array}$ & $\begin{array}{l}\text { Seasonal/ } \\
\text { situational }\end{array}$ & Literature $^{\mathrm{i}}$ \\
\hline $\begin{array}{l}\text { Struthio camelus } \\
\text { dom. }\end{array}$ & 2.8 & Polygyn & Bipar & High & $P$ & 60 & - & Dispersed & Mm groups & Seasonal & $\mathrm{C}$ & Seasonal & Degen et al., 1994 \\
\hline $\begin{array}{l}\text { Dromaius } \\
\quad \text { novaehollandiae }\end{array}$ & 2.8 & Monog & Patern & Low & $\mathrm{P}$ & 100 & - & Dispersed & Solitary & Seasonal & $\mathrm{C}$ & Seasonal & Malecki et al., 1998 \\
\hline Apteryx australis & 47.6 & Monog & Patern & Low & $\mathrm{P}$ & 100 & - & Dispersed & Solitary & Long-term & $\mathrm{F}$ & Seasonal & Cockrem and Potter, 1990* \\
\hline $\begin{array}{l}\text { Colinus virginianus } \\
\text { texanus }\end{array}$ & 60.3 & Monog & Patern $^{\mathrm{c}}$ & Low & $\mathrm{P}$ & 100 & - & & Mm groups & Seasonal & $\mathrm{C}$ & Situational & Vleck and Dobrott, 1993 \\
\hline Tetrao tetrix & 2.5 & Promisc & Matern & High & $\mathrm{P}$ & 0 & - & Dispersed & Mm groups & None & $\mathrm{F}$ & Seasonal & Alatalo et al., 1996 \\
\hline Lagopus lagopus & 17.2 & Monog & Bipar & High & $\mathrm{P}$ & 0 & - & Dispersed & Solitary & Seasonal & $\mathrm{F}$ & Seasonal & Hannon and Wingfield, 1990* \\
\hline $\begin{array}{c}\text { Lagopus mutus } \\
\text { hyperboreus }\end{array}$ & 3.7 & Polygyn & Bipar & High & $\mathrm{P}$ & 0 & - & Dispersed & Solitary & Seasonal & $\mathrm{F}$ & Seasonal & Stokkan and Sharp, 1990* \\
\hline Meleagris gallopavo & 2.7 & Polygyn & Matern & High & $P$ & 0 & - & Aggregated & Solitary & None & $\mathrm{C}$ & Seasonal & Lisano and Kennamer, 1977* \\
\hline $\begin{array}{l}\text { Phasianus colchicus } \\
\text { versicolor }\end{array}$ & 1.6 & Polygyn & Matern & High & $\mathrm{P}$ & 0 & - & Dispersed & Mm groups & None & $\mathrm{C}$ & Seasonal & Sakai and Ishii, 1986* \\
\hline Coturnix c. japonica & 1.2 & Polygyn & Matern & High & $\mathrm{P}$ & 0 & - & & Solitary & Seasonal & $\mathrm{C}$ & Seasonal & Ishii et al., 1994 \\
\hline Alectoris rufa & 9.7 & Monog & Bipar & High & $P$ & 30 & - & Dispersed & Solitary & Long-term & $\mathrm{C}$ & Seasonal & Bottoni et al., 1993 \\
\hline Perdix perdix & 14.9 & Monog & Bipar & Low & $\mathrm{P}$ & 0 & - & Dispersed & Mm groups & Long-term & $\mathrm{F}$ & Seasonal & Fraissinet et al., 1987* \\
\hline Branta canadensis & 3.6 & Monog & Bipar & High & $\mathrm{P}$ & 0 & - & Aggregated & $\mathrm{Mm}$ groups & Long-term & $\mathrm{C}$ & Situational & Akesson and Raveling, 1981* \\
\hline Anser anser & 3.4 & Monog & Bipar & High & $\mathrm{P}$ & 0 & - & Dispersed & $\mathrm{Mm}$ groups & Long-term & $\mathrm{F}$ & Seasonal & Hirschenhauser et al., $1999 * *$ \\
\hline Anser anser dom. & 1.5 & Polygyn & Bipar & High & $\mathrm{P}$ & 0 & - & Dispersed & Mm groups & Long-term & $\mathrm{C}$ & Seasonal & Kotrschal et al., $2000^{* * *}$ \\
\hline Anser indicus & 6.0 & Monog & Bipar & High & $\mathrm{P}$ & 0 & - & Dispersed & $\mathrm{Mm}$ groups & Long-term & $\mathrm{F}$ & Seasonal & Dittami, 1981* \\
\hline $\begin{array}{l}\text { Somateria } \\
\quad \text { mollissima }\end{array}$ & 3.1 & Polygyn & Matern & High & $\mathrm{P}$ & 0 & - & Aggregated & Mm groups & Seasonal & $\mathrm{F}$ & Seasonal & Gorman, 1977 \\
\hline Anas platyrhynchus & 1.6 & Polygyn & atern & High & $\mathrm{P}$ & 0 & - & ggregated & Mm groups & Long-term & $\mathrm{C}$ & Seasonal & Paulke and Haase, 1978* \\
\hline $\begin{array}{l}\text { Anas platyrhynchus } \\
\text { dom. }\end{array}$ & 2.1 & Polygyn & Matern & High & $\mathrm{P}$ & 0 & - & Aggregated & Mm groups & Long-term & $\mathrm{C}$ & Seasonal & Paulke and Haase, 1978* \\
\hline Picoides borealis & 13.1 & Monog & Bipar $^{\mathrm{d}}$ & Low & A & 30 & 37 & ggregated & Mm groups & Long-term & $\mathrm{F}$ & ssonal & Khan et \\
\hline Ceryle rudis & 2.7 & Monog & Bipar $^{\mathrm{d}}$ & High & A & 60 & 60 & Aggregated & Mm groups & Seasonal & $\mathrm{F}$ & Seasonal & Reyer et al., 1986 \\
\hline Psittacula krameri & 2.5 & Monog & Bipar & High & A & 0 & 50 & Aggregated & Mm groups & Seasonal & $\mathrm{F}$ & Seasonal & Krishnapradasart et al., 1988 \\
\hline $\begin{array}{l}\text { Streptopelia } \\
\text { decaocto }\end{array}$ & 9.7 & Monog & Bipar & Mod & A & 33 & 50 & Dispersed & Mm groups & Long-term & $\mathrm{C}$ & Seasonal & Péczely and Pethes, $1979^{*}$ \\
\hline Streptopelia risoria & 6.2 & Monog & Bipar & Mod & A & 50 & 50 & Dispersed & & Long-term & $\mathrm{C}$ & Situational & O'Connel et al., 1981 \\
\hline $\begin{array}{l}\text { Chlamydotis } \\
\text { macqueenii }\end{array}$ & 5.1 & Monog & Matern & Mod & $\mathrm{P}$ & 0 & - & Dispersed & Mm groups & Seasonal & $\mathrm{C}$ & Seasonal & Saint Jalme et al., 1996 \\
\hline $\begin{array}{l}\text { Chlamydotis } \\
\text { undulata }\end{array}$ & 3.2 & Promisc & Matern & Mod & $P$ & 0 & - & Dispersed & Mm groups & None & $\mathrm{C}$ & Seasonal & Saint Jalme et al., 1996 \\
\hline Phalaropus lobatus & 32.4 & Polyandr & Patern & Low & $\mathrm{P}$ & 100 & - & ispersed & Solitary & onal & $\mathrm{F}$ & nal & Gratto-Trevor et al., 1990* \\
\hline Phalaropus tricolor & 5.9 & Polyandr & Patern & Mod & $P$ & 100 & - & ispersed & Solitary & Seasonal & $\mathrm{F}$ & Seasonal & Fivizzani et al., 1986* \\
\hline Actitis macularia & 5.3 & Polyandr & Patern & Mod & $\mathrm{P}$ & 100 & - & ispersed & Solitary & Seasonal & $\mathrm{F}$ & sonal & Fivizzani and Oring. 1986* \\
\hline Calidris pusilla & 16.3 & Monog & Bipar & High & $P$ & 50 & - & ispersed & $\mathrm{Mm}$ groups & Seasonal & $\mathrm{F}$ & sonal & Gratto-Trevor et al., 1990* \\
\hline Chionis minor & 13.5 & Monog & Bipar & Mod & A & 0 & 50 & Dispersed & Mm groups & Long-term & $\mathrm{F}$ & Seasonal & Burger and Millar, 1980* \\
\hline Larus occidentalis & 14.0 & Monog & Bipar & Mod & A & 42 & 57 & Aggregated & Mm groups & Long-term & $\mathrm{F}$ & Seasonal & Wingfield, 1990* \\
\hline Falco tinnunculus & 12.6 & Monog & Bipar & High & A & 0 & 90 & Dispersed & Solitary & Long-term & $\mathrm{F}$ & Seasonal & Meijer and Schwabl, 1989* \\
\hline $\begin{array}{l}\text { Parabuteo } \\
\text { unicinctus }\end{array}$ & 22.2 & Monog & Bipar $^{\mathrm{d}}$ & Low & A & 30 & 50 & Aggregated & Mm groups & Long-term & $\mathrm{F}$ & Seasonal & Mays et al., $1991^{*}$ \\
\hline Phaeton rubricauda & 5.7 & of & par & $\mathrm{H}$ & A & J & 50 & & & & $\mathrm{~F}$ & & 00 \\
\hline Sula dactylatra & 16.0 & Monog & Bipar & High & A & 50 & 50 & gregated & $\mathrm{Mm}$ groups & Long-term & $\mathrm{F}$ & nal & née et al., 2000 \\
\hline Sula sula & 4.0 & Monog & Bipar & High & A & 50 & 50 & ggregated & Mm groups & Long-term & $\mathrm{F}$ & & et al., 2000 \\
\hline Sula nebouxii & 6.4 & Monog & Bipar & High & A & 50 & 50 & gragated & Mm groups & Long-term & $\mathrm{F}$ & onal & $d$ et al., $1999 b$ \\
\hline $\begin{array}{l}\text { Phalacrocorax } \\
\text { capensis }\end{array}$ & 1.8 & Monog & Bipar & High & A & 50 & 50 & Aggregated & Mm groups & Seasonal & $\mathrm{F}$ & Seasonal & Berry et al., $1979^{*}$ \\
\hline $\begin{array}{l}\text { Eudyptes } \\
\quad \text { chrysolophus }\end{array}$ & 6.4 & Monog & Bipar & Low & A & 50 & 50 & Aggregated & Mm groups & Long-term & $\mathrm{F}$ & Seasonal & Williams, 1992* \\
\hline $\begin{array}{r}\text { Megadyptes } \\
\text { antipodes }\end{array}$ & 14.1 & Monog & Bipar & Low & A & 50 & 50 & Aggregated & Solitary & Long-term & $\mathrm{F}$ & Seasonal & Cockrem and Seddon, 1994 \\
\hline Pygoscelis adeliae & 9.2 & $\operatorname{nog}$ & B & & A & 63 & 5 & $d$ & ps & $\mathrm{m}$ & $\mathrm{F}$ & & 1., $1986 *$ \\
\hline Pygoscelis papua & 21.2 & Monog & & & A & 50 & 50 & & $\mathrm{Mmg}$ & erm & $\mathrm{F}$ & & ms, 1992* \\
\hline Aptenodytes forsteri & 16.4 & Monog & Bipar & Low & A & 100 & 50 & Aggregated & $\mathrm{Mm}$ groups & Long-term & $\mathrm{F}$ & Seasonal & Groscolas et al., 1986* \\
\hline $\begin{array}{l}\text { Aptenodytes } \\
\text { patagonicus }\end{array}$ & 24.8 & Monog & Bipar & Low & A & 50 & 50 & Aggregated & Mm groups & Long-term & $\mathrm{F}$ & Seasonal & Mauget et al., 1994* \\
\hline $\begin{array}{l}\text { Diomedea } \\
\text { chrysostoma }\end{array}$ & 15.0 & Monog & Bipar & Low & A & 50 & 50 & Aggregated & Mm groups & Long-term & $\mathrm{F}$ & Seasonal & Hector et al., 1986a* \\
\hline Diomedea exulans & 5. & $\mathrm{M}$ & B & & A & 5 & 5 & ed & $\mathrm{N}$ & & $\mathrm{F}$ & & \\
\hline $\begin{array}{l}\text { Diomedea } \\
\quad \text { melanophris }\end{array}$ & 27.8 & Monog & Bipar & Low & A & 50 & 50 & Aggregated & Mm groups & Long-term & $\mathrm{F}$ & Seasonal & Hector et al., 1986a* \\
\hline $\begin{array}{l}\text { Hylophylax } \\
\text { naevioides }\end{array}$ & 19.4 & Monog & Bipar & High & $A^{P}$ & 50 & 50 & ed & ary & -term & $\mathrm{F}$ & nal & ., 1999 \\
\hline $\begin{array}{l}\text { Ptilonorhynchus } \\
\text { violaceus }\end{array}$ & 2.18 & Promisc & Matern & High & $\mathrm{A}^{\mathrm{P}}$ & 0 & 0 & Dispersed & Solitary & None & $\mathrm{F}$ & Situational & Borgia and Wingfield, 1991* \\
\hline Malurus cyaneus & 2.8 & Monog & Bipar $^{\mathrm{e}}$ & High & $P$ & 0 & 50 & Aggregated & Mm groups & Seasonal & $\mathrm{F}$ & nal & $\begin{array}{l}\text { Peters, } 2000 \\
\quad \text { (continued on } n\end{array}$ \\
\hline
\end{tabular}


Table 2 (continued)

\begin{tabular}{|c|c|c|c|c|c|c|c|c|c|c|c|c|c|}
\hline Species & $\mathrm{AR}$ & Mating $^{\mathrm{a}}$ & $\begin{array}{l}\text { Parental } \\
\text { care }^{\text {b }}\end{array}$ & $\begin{array}{l}\text { Territory } \\
\text { aggress. }^{f}\end{array}$ & $\begin{array}{l}\text { Precocial/ } \\
\text { altricial }^{\mathrm{g}}\end{array}$ & INC\% & $\mathrm{FO} \%$ & $\begin{array}{l}\text { Breeding } \\
\text { density }\end{array}$ & $\begin{array}{l}\text { Group } \\
\text { livingh }\end{array}$ & Pairbond & $\begin{array}{l}\text { Free- } \\
\text { living/ } \\
\text { captive }\end{array}$ & $\begin{array}{l}\text { Seasonal/ } \\
\text { situational }\end{array}$ & Literature $^{\mathrm{i}}$ \\
\hline Lanius collurio & 8.4 & Monog & Bipar & High & $\mathrm{A}^{\mathrm{P}}$ & 0 & 56 & Dispersed & Solitary & Seasonal & $\mathrm{F}$ & Seasonal & Fornasari et al., 1992* \\
\hline $\begin{array}{c}\text { Pachycephala } \\
\text { rufiventris }\end{array}$ & 19.8 & Monog & Bipar & High & $\mathrm{A}^{\mathrm{P}}$ & 36.2 & 50 & & & & $\mathrm{~F}$ & Situational & McDonald et al., 2001 \\
\hline Corvus frugilegus & 6.1 & Monog & Bipar & Mod & $\mathrm{A}^{\mathrm{P}}$ & 0 & 69 & Aggregated & Mm groups & Long-term & $\mathrm{F}$ & Seasonal & Péczely and Pethes, $1982 *$ \\
\hline $\begin{array}{l}\text { Aphelocoma } \\
\text { coerulescens }\end{array}$ & 7.5 & Monog & Bipar $^{\mathrm{d}}$ & Mod & $\mathrm{A}^{\mathrm{P}}$ & 0 & 60 & Dispersed & Mm groups & Long-term & $\mathrm{F}$ & Seasonal & Schoech et al., 1991* \\
\hline $\begin{array}{l}\text { Aphelocoma } \\
\text { californica }\end{array}$ & 9.1 & Monog & Bipar & Mod & $\mathrm{A}^{\mathrm{P}}$ & 0 & 50 & Dispersed & Mm groups & Long-term & $\mathrm{F}$ & Seasonal & Vleck and Brown, 1999 \\
\hline $\begin{array}{l}\text { Aphelocoma } \\
\text { ultramarina }\end{array}$ & 2.5 & Polygyn & Bipar $^{\mathrm{e}}$ & High & $\mathrm{A}^{\mathrm{P}}$ & 0 & 50 & Dispersed & Mm groups & Long-term & $\mathrm{F}$ & Seasonal & Vleck and Brown, 1999 \\
\hline Cinclus pallasii & 4.9 & Monog & Bipar & High & $\mathrm{A}^{\mathrm{P}}$ & 0 & 50 & Dispersed & Solitary & Long-term & $\mathrm{F}$ & Seasonal & Kofuji et al., $1993^{* *}$ \\
\hline Turdus merula & 4.8 & Monog & Bipar & Mod & $\mathrm{A}^{\mathrm{P}}$ & 0 & 51 & Dispersed & Solitary & Seasonal & $\mathrm{F}$ & Seasonal & Schwabl et al., 1980* \\
\hline Saxicola torquata & 3.8 & Polygyn & Bipar & High & $\mathrm{A}^{\mathrm{P}}$ & 0 & 50 & Dispersed & Solitary & Seasonal & $\mathrm{F}$ & Situational & Gwinner et al., 1994 \\
\hline Ficedula hypoleuca & 7.7 & Polygyn & Bipar & Mod & $\mathrm{A}^{\mathrm{P}}$ & 0 & 44 & Dispersed & Solitary & Seasonal & $\mathrm{F}$ & Seasonal & Silverin and Wingfield, 1982* \\
\hline $\begin{array}{l}\text { Lamprotornis } \\
\text { chalybaeus }\end{array}$ & 2.2 & Monog & Bipar & High & $\mathrm{A}^{\mathrm{P}}$ & $?$ & $?$ & & & & $\mathrm{~F}$ & Seasonal & Dittami and Gwinner, 1990 \\
\hline Sturnus vulgaris & 10.4 & Polygyn & Bipar & Mod & $\mathrm{A}^{\mathrm{P}}$ & 24 & 50 & Aggregated & Mm groups & Seasonal & $\mathrm{F}$ & Seasonal & Ball and Wingfield, 1987* \\
\hline $\begin{array}{l}\text { Toxostoma } \\
\text { curvrostre }\end{array}$ & 6.5 & Monog & Bipar & High & $\mathrm{A}^{\mathrm{P}}$ & 75 & 50 & & & Long-term & $\mathrm{F}$ & Seasonal & Vleck, 1993* \\
\hline $\begin{array}{l}\text { Campylorhynchus } \\
\text { brunneicapillus }\end{array}$ & 6.7 & Monog & Bipar & High & $\mathrm{A}^{\mathrm{P}}$ & 0 & 50 & Dispersed & Solitary & Seasonal & $\mathrm{F}$ & Seasonal & Vleck, 1993* \\
\hline Parus major & 7.7 & Monog & Bipar & Mod & $\mathrm{A}^{\mathrm{P}}$ & 40 & 50 & Dispersed & Mm groups & Seasonal & $\mathrm{F}$ & Seasonal & Silverin, 1990 \\
\hline $\begin{array}{c}\text { Acrocephalus } \\
\text { scirpaceus }\end{array}$ & 2.7 & Monog & Bipar & High & $\mathrm{A}^{\mathrm{P}}$ & 12 & 50 & Dispersed & Solitary & Seasonal & $\mathrm{F}$ & Seasonal & Dittami et al., 1991 \\
\hline Cettia diphone & 2.9 & Polygyn & Matern & High & $\mathrm{A}^{\mathrm{P}}$ & 0 & 0 & Dispersed & Solitary & None & $\mathrm{F}$ & Seasonal & Wada et al., 1999 \\
\hline Passer domesticus & 4.6 & Monog & Bipar & High & $\mathrm{A}^{\mathrm{P}}$ & 39 & 45 & Aggregated & Mm groups & Long-term & $\mathrm{F}$ & Seasonal & Hegner and Wingfield, 1986* \\
\hline Passer rufocinctus & 2.4 & Monog & Bipar & Mod & $\mathrm{A}^{\mathrm{P}}$ & $?$ & $?$ & & & & $\mathrm{~F}$ & Seasonal & Dittami and Gwinner, 1990 \\
\hline $\begin{array}{l}\text { Lonchura striata } \\
\text { dom. }\end{array}$ & 19.5 & Monog & Bipar & Low & $\mathrm{A}^{\mathrm{P}}$ & 50 & 50 & & Mm groups & & $\mathrm{C}$ & Seasonal & Seiler et al., 1992 \\
\hline Plocepasser mahali & 4.9 & Monog & Bipar $^{\mathrm{e}}$ & Mod & $\mathrm{A}^{\mathrm{P}}$ & 25 & 50 & & Mm groups & Long-term & $\mathrm{C}$ & Seasonal & Levin and Wingfield, 1992 \\
\hline Ploceus reichenowi & 3.3 & Monog & Bipar & Mod & $\mathrm{A}^{\mathrm{P}}$ & 0 & 50 & & Mm groups & Long-term & $\mathrm{F}$ & Seasonal & Dittami and Gwinner, 1990 \\
\hline Ploceus philippinus & 2.2 & Polygyn & Matern & High & $\mathrm{A}^{\mathrm{P}}$ & 0 & 0 & Aggregated & Mm groups & Seasonal & $\mathrm{F}$ & Seasonal & Narasimhacharya et al., 1980 \\
\hline $\begin{array}{l}\text { Carpodacus } \\
\text { mexicanus }\end{array}$ & 6.4 & Polygyn & Bipar & High & $\mathrm{A}^{\mathrm{P}}$ & 0 & 30 & Dispersed & Solitary & Seasonal & $\mathrm{C}$ & Situational & Belthoff and Dufty, 1994 \\
\hline Agelaius phoeniceus & 1.2 & Polygyn & Matern & High & $\mathrm{A}^{\mathrm{P}}$ & 0 & 5 & Dispersed & Mm groups & None & $\mathrm{F}$ & Seasonal & Beletsky et al., 1989* \\
\hline Molothrus ater & 2.1 & Monog & - & High & $-^{P}$ & 0 & 0 & Aggregated & Mm groups & None & $\mathrm{F}$ & Seasonal & Dufty, 1993* \\
\hline Melospiza melodia & 12.4 & Monog & Bipar & High & $\mathrm{A}^{\mathrm{P}}$ & 0 & 71 & Dispersed & Mm groups & Seasonal & $\mathrm{F}$ & Seasonal & Wingfield, 1994 \\
\hline $\begin{array}{l}\text { Zonotrichia } \\
\text { leucophrys }\end{array}$ & 3.2 & Monog & Bipar & Mod & $\mathrm{A}^{\mathrm{P}}$ & 0 & 40 & Dispersed & Solitary & Seasonal & $\mathrm{F}$ & Seasonal & Moore, 1982 \\
\hline Junco hyemalis & 4.8 & Monog & Bipar & High & $\mathrm{A}^{\mathrm{P}}$ & 0 & 50 & & $\mathrm{Mm}$ groups & Seasonal & $\mathrm{F}$ & Seasonal & Chandler et al., 1997 \\
\hline Amphispiza bilineata & 12.4 & Monog & Bipar & High & $\mathrm{A}^{\mathrm{P}}$ & $?$ & 50 & & Mm groups & & $\mathrm{F}$ & Seasonal & Vleck, 1993* \\
\hline Spizella arborea & 21.0 & Monog & Bipar & Mod & $\mathrm{A}^{\mathrm{P}}$ & 0 & 50 & Dispersed & Mm groups & Seasonal & $\mathrm{F}$ & Seasonal & Hunt et al., 1995* \\
\hline $\begin{array}{l}\text { Calcarius } \\
\quad \text { lapponicus }\end{array}$ & 6.8 & Monog & Bipar & Mod & $\mathrm{A}^{\mathrm{P}}$ & 0 & 50 & Dispersed & $\mathrm{Mm}$ groups & Seasonal & $\mathrm{F}$ & Seasonal & Hunt et al., 1995* \\
\hline $\begin{array}{l}\text { Plectrophenax } \\
\quad \text { nivalis }\end{array}$ & 4.9 & Monog & Bipar & High & $\mathrm{A}^{\mathrm{P}}$ & $?$ & 50 & Aggregated & Solitary & Seasonal & $\mathrm{F}$ & Seasonal & Romero et al., 1998 \\
\hline
\end{tabular}

Note. INC, male's contribution to incubation; FO, male's contribution to feeding offspring.

${ }^{a}$ Polygn, polygynous ( $>5 \%$ polygyny); Monog, monogamous ( $<5 \%$ polygyny); Promisc, promiscuous.

${ }^{\mathrm{b}}$ Bipar, biperental care; Patern, paternal care; Matern, maternal care.

${ }^{\mathrm{c}}$ Study on foster-fathering males. Note that bobwhite quails usually share incubation duties.

${ }^{\mathrm{d}}$ Only male helpers.

${ }^{\mathrm{e}}$ Helpers from either sex.

${ }^{\mathrm{f}}$ High, highly territorial breeding males; Mod, moderately.

${ }^{\mathrm{g}} \mathrm{A}^{\mathrm{P}}$, (altrical) passerine species.

${ }^{\mathrm{h}} \mathrm{Mm}$ groups, multimale groups.

*, in Wingfield et al., 2000 sample; **, data derived from fecal equivalents.

season [species with solitary males $(N=26)$ or species with multimale groups $(N=52)$, Møller and Birkhead, 1993]. The effect of the pairbond type on the specific androgen response rates was also qualitatively determined as "no pairbond" $(N=7)$, "seasonal bond" $(N=32)$, or "longterm bond" $(N=39)$ and was coded with dummy variables as the degree of paternal care. Finally, the precociality of the species was taken into account [altricial $(N=58)$ vs pre- cocial species $(N=25)$ ]. Numbers of species may vary depending on the information available.

\section{Results}

Plots of the potential AR from the 84 sampled bird species by the ratio d confirmed the pattern observed by 


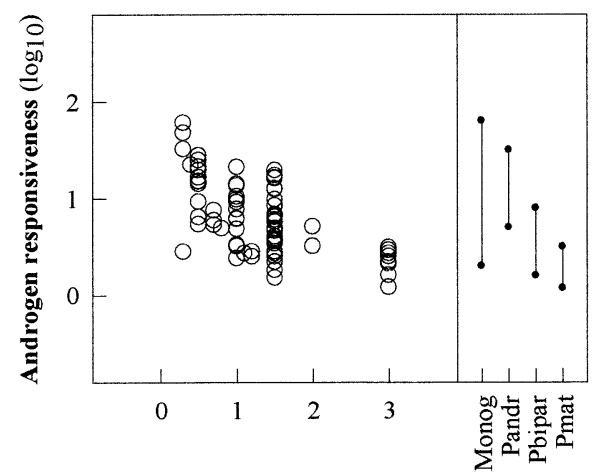

Ratio d

Mating system

Fig. 1. Left panel: interspecific pattern of the AR (ratio $[\mathrm{C}-\mathrm{A}] /[\mathrm{B}-\mathrm{A}]$; Wingfield et al., 1990) as determined from the literature among males of 84 bird species plotted by the ratio d (i. e., male-male aggressiveness divided by the degree of paternal care). Right panel: ranges of AR in males from monogamous (Monog), polyandrous (Pandr), polygynous and biparental (Pbipar), and polygynous and maternal (Pmat) species. For references of androgen data sources please refer to Table 2 .

Wingfield et al. (1990, 2000) with an increased sample size. That is, males from species with a high degree of paternal care seemed to respond to social challenges with larger androgen increases than species with high male-male aggressiveness and a low degree of paternal investment (Fig. 1). In order to test the effects of each of the parameters involved into the ratio d, the independent variables malemale aggressiveness, degree of paternal care, and mating system were entered into a regression analysis of all observed AR rates. The multiple effect of all variables together satisfied the predicted variation of androgen rates within confidence intervals of $\pm 4 \%\left(F_{7.76}=25.96 ; P<0.0001\right)$. So far, the results could be interpreted as male-male aggressiveness and paternal care contributing the strongest effects on the observed interspecific variation of AR, with polygyny providing only a minor additional explanation.

\section{Control for phylogeny}

To control for the effect of phylogenetic relatedness, the independent variables male-male aggressiveness, mating strategy, and degree of paternal care were entered into single and multiple regression analyses (GLS) by comparing the results from using raw data with those from using phylogenetic contrasts. The single effects of mating system and male-male aggressiveness on AR remained significant after adding the phylogenetic information (Fig. 2a and b). Additionally, to rule out potential bias due to the assumption of equidistances between the different phenotypes of a continuous scale for the different mating systems, we compared the AR of males from monogamous species (mean AR \pm SEM: $11.18 \pm 1.34$ ) with those from polygynous species (mean AR \pm SEM: $3.37 \pm 0.57$; discrete variables that imply no order of the independent variables). Also by using this approach, the effect of mating system on AR remained robust to incorporating information on the phylogenetic relationship among the species included (without phylogeny: $F_{1,76}=24.56 ; P<0.0001$; with phylogeny: $\left.F_{1,76}=22.28 ; P<0.0001\right)$. The effect of the degree of paternal care on AR also remained significant (Fig. 2c). However, both analyses (without and with phylogeny) showed that the major variation of AR was explained by the change from no paternal care to at least some paternal care (biparental), whereas the effect of the change from biparental to full paternal care did not remain significant (Fig. 2c).
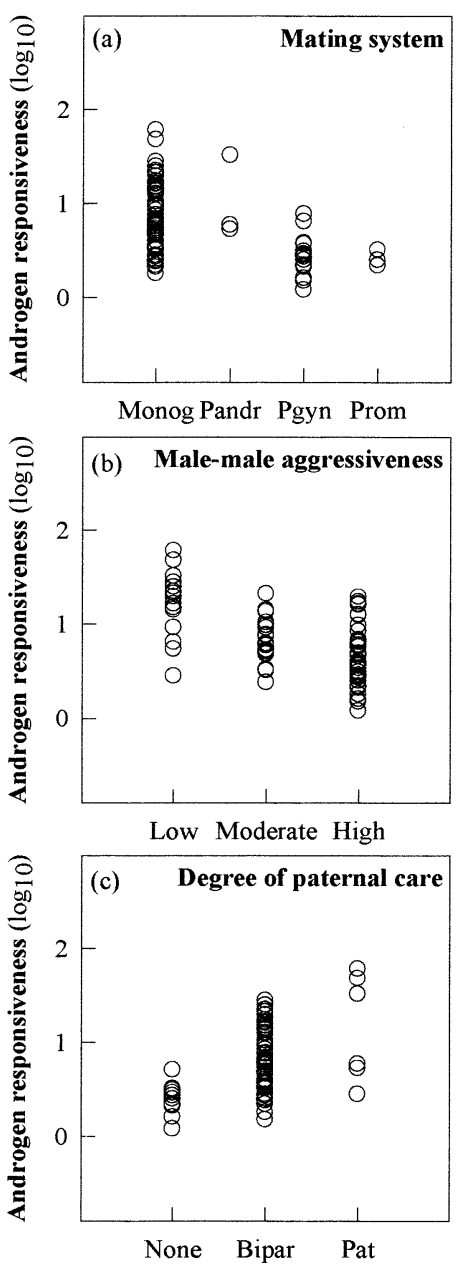

Fig. 2. Interspecific patterns of the AR (please refer to legend of Fig. 1 for details) by (a) mating system (Monog, monogamous; Pandr, polyandrous; Pgyn, polygynous; Prom, promiscuous), (b) male-male aggressiveness, and (c) by the degree of paternal care, (None, no paternal care; Bipar, biparental; Pat, paternal care.) The single effects of mating system (without phylogeny: $F_{3,80}=9.91 ; P<0.0001$; with phylogeny: $F_{3,80}=7.37 ; P<$ 0.0005 ), male-male aggressiveness (without phylogeny: $F_{2,81}=21.34 ; P$ $<0.0001$; with phylogeny: $\left.F_{2,81}=16.34 ; P<0.0001\right)$ and the degree of paternal care (without phylogeny: $F_{2,81}=15.08 ; P<0.0001$; with phylogeny: $F_{2,81}=15.08 ; P<0.0001$ ) on AR remained significant after adding the phylogenetic information. However, in the case of paternal care, both analyses (without and with phylogeny) revealed that the major variation of AR was explained by the change from no paternal care to biparental care $\left(F_{1,82}=25.95 ; P<0.0001\right)$, whereas the effect of the change from biparental to full paternal care did not remain significant $\left(F_{1,82}=\right.$ 4.28; $P>0.05$ ). 

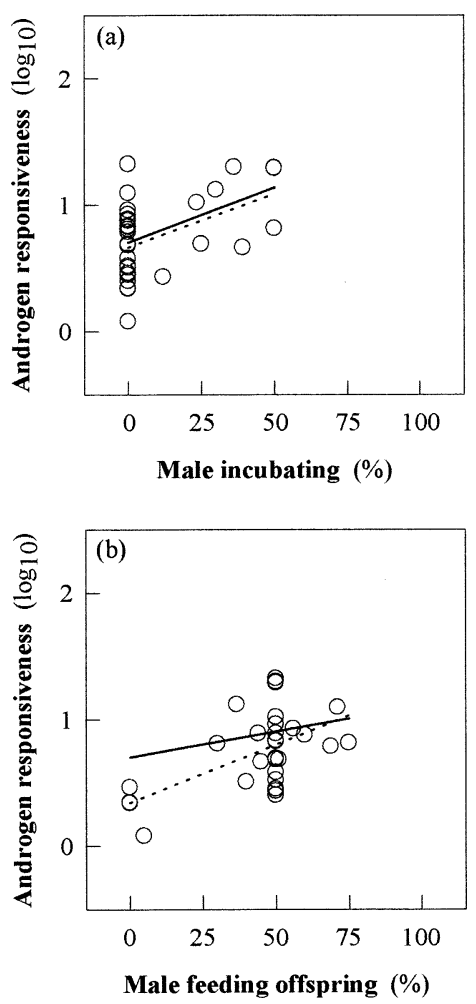

Fig. 3. Interspecific patterns of the AR (please refer to legend of Fig. 1 for details) among males of 32 passerine bird species by the contribution of the male parent during (a) incubation (without phylogeny: $F_{1,30}=7.97 ; P<$ 0.01 ; with phylogeny: $F_{1,30}=7.16 ; P<0.02$ ) and during (b) feeding the offspring (without phylogeny: $F_{1.30}=16.29 ; P<0.0005$ : with phylogeny: $\left.F_{1,30}=1.46 ; P>0.05\right)$. Broken line represents the single regression on raw data; solid line represents the single regression including phylogenetic distances (GLS). Only the single effect of the males' contribution to incubation remained significant after control for phylogeny, whereas the effect of male chick feeding did not.

In line with this, there was a highly significant effect of all independent variables on the observed androgen responses, with a nonsignificant contribution of paternal care. Thus, the final model, which is also the best acording to Akaike's information criterium that takes the number of predictors into account (Efron and Tibshirani, 1993, Tabachnick and Fidell, 2001) included male-male aggressiveness and mating system only $\left(F_{7,76}=5.69 ; P<0.0001\right)$ and suggested (as determined by the confidence limits) that only male-male aggressiveness and mating strategy were relevant for evolutionary changes of the AR.

\section{Paternal investment among passerine species}

Among the raw data of the subsample of passerine species $(N=32)$, the effects of incubation and feeding offspring were significant (Fig. $3 \mathrm{a}$ and $\mathrm{b}$ ). Controlling for phylogeny within this group, however, only the single effect of the males' contribution to incubation was significant (Fig. 3a), while chick feeding was not (Fig. 3b). This demonstrated that the major variation of AR was due to the evolutionary change from no male incubation to male incubation, rather than to the males' involvement during phases of feeding offspring between hatching and fledging.

\section{Social environment}

The observed androgen response rates were not different due to group living, either between species with dispersed breeding or those with breeding aggregations $\left(F_{1,71}=\right.$ $0.008 ; P>0.05)$, or between species with solitarily living males or those with multimale groups $\left(F_{1,77}=0.006 ; P>\right.$ 0.05). Males from species with either type of pairbond showed larger androgen response rates than males from species with no pairbond at all $\left(F_{1,77}=7.91 ; P<0.01\right)$. Response rates of altricial and of precocial species were not significantly different $\left(F_{1,82}=0.95 ; P>0.05\right)$.

\section{Discussion}

The challenge hypothesis proposes an interaction among mating strategy, the degree of paternal investment, territorial male-male aggressiveness, and the males' potential AR to social challenges at both the intraspecific and the interspecific levels (Wingfield et al., 1990, 2000). This study has focused on the predictions of the challenge hypothesis at the interspecific level.

The interspecific patterns described by Wingfield and co-workers remained relatively robust in our analysis with an enlarged sample size of avian species (Fig. 1). Because only a minor fraction of the sample originated from situational data, i.e., provided a classification of the male-male aggressiveness from simulated territorial intruder experiments (Wingfield, 1990; Table 2), we observed difficulties to reproduce the ratio d (i.e., male-male aggressiveness divided by the degree of paternal investment). In order to apply multivariate statistics, the effects of the different parameters (mating strategy, degree of paternal care, and male-male aggressiveness) were investigated separately.

Controlling for the phylogenetic relatedness (Felsenstein, 1985; Martins and Hansen, 1997; Pagel and Harvey, 1988) among all of the sampled species revealed that predominantly mating system and male-male aggressiveness were relevant for an evolutionary change of the AR. Also, repeating analyses with discrete variables for mating system (monogamous vs polygynous) confirmed these results. The effect of the degree of paternal care (categories as in Wingfield et al., 1990, 2000; Table 2) on the interspecific patterns of AR, however, was dramatically reduced. To check for the lack of paternal care effects we then repeated analyses of the effects of paternal investment using a quantified scale of male incubation and feeding offspring rates among the subsample of closely related passerine (altricial) species $(N=32)$. Results from this subsample revealed significant effects of the males' specific contribution during incubation on $\mathrm{AR}$, but no effect of feeding offspring rates 
(Fig. 3a and b). Although this indicated that paternal incubation seemed to interact with AR to some degree, the effect was observed only among a subsample of altricial species and may not have covered a representative fraction of the overall variability of mating systems (Table 2). Therefore, we consider the results originating from comparisons among the total sample (including precocial species) as generally more essential.

Although each of the approaches presented in this study has its own methodological flaw, they all result in the same robust pattern. It suggests that differences of AR have clearly evolved in response to variation in mating strategies and male-male aggression, whereas changes of AR in response to altered degrees of paternal investment appeared more complex. Among the subsample of the closely related passerines, only the change from no male incubation to male shares incubation contributed significantly to the evolution of different AR in response to territorial challenges. Therefore, the males' contribution during incubation may be regarded as the maximum paternal investment during all breeding phases, which was also reflected in the observed AR. This conclusion may sound contradictory to a large body of research that has shown a modulator role of testosterone in the trade-off between male-male aggressiveness and paternal care (specifically measuring feeding offspring rates) at the intraspecific level (Silverin, 1980; Wingfield, 1984b; Hegner and Wingfield, 1987; Beletsky et al., 1989; Dittami et al., 1991; Ketterson and Nolan, 1992; De Ridder et al., 2000), although several recent studies have suggested that low androgen levels are not a requirement for paternal behavior in all species (Hunt et al., 1999; Van Duyse et al., 2000, 2002; Lynn et al., 2002). However, the observed interactions between social environment and androgen responses do not have to act equally at both levels of analysis. Hence, the nonsignificant effect of the degree of paternal care in general on the flexibility of male AR using data controlled for phylogeny, stresses the importance of precisely adding the operational unit to the proposed social modulation of androgen levels in birds.

The trade-off between territorial male-male aggression and paternal care, of which flexible androgen patterns are the underlying mechanism, is defined for the fitness costs of mutually exclusive life-history decisions/states at an individual level (Stearns, 1989; Ketterson and Nolan, 1992). This trade-off was reflected in the presented interspecific comparisons as well. In the attempt to test for the basic trade-off assumptions of the challenge hypothesis, we observed a nonrandom pattern of male-male aggressiveness and paternal care (Table 3). Thus, it may be correct to consider the antagonistic nature of male-male aggressiveness and paternal care even at the interspecific level. Consequently, we may have observed the disappeared effect of paternal care on differences in AR independently of the seemingly remaining antagonistically interacting parameters.

The challenge hypothesis (Wingfield et al., 1990) also
Table 3

The trade-off between male-male aggressiveness and degree of paternal care among males from 84 bird species

\begin{tabular}{lcll}
\hline Mm aggressiveness & Maternal & Biparental & Paternal \\
\hline Low & 0 & 13 & 4 \\
Moderate & 2 & 17 & 2 \\
High & 12 & 34 & 0 \\
\hline
\end{tabular}

Note. Table shows the numbers of species per trait combination. Low male-male aggressiveness was observed exclusively among males with any paternal investment (biparental or paternal). In contrast, high malemale aggressiveness was observed predominantly among males from biparental and maternal species, but not among males from species with full paternal care.

offers a conceptual framework for attempts to explain the plasticity and the predictability of male androgen responses with parameters of the social environment, such as breeding dispersal, group living, social status (Beletsky et al., 1992; Wada et al., 1999), alternative life histories (Moore, 1991; Ketterson and Nolan, 1992; Oliveira et al., 2001b), and the predictability of the physical environment (Wingfield et al., 1993, 2000). Because species living at high group densities may be adapted to cope with higher frequencies of malemale interactions, the challenge hypothesis would predict higher basal androgen levels throughout the breeding phases and hence, smaller androgen increases in response to an additional social challenge as compared with solitary males (Beletsky et al., 1992; Pankhurst and Barnett, 1993; Wada et al., 1999; Wingfield et al., 1999a). Among the species included in the present study there was no effect either of the breeding dispersal (i.e., species with dispersed or aggregated nesting; Westneat and Sherman, 1997; Searcy et al., 1999) or of group living outside of the breeding season (i.e., species with solitary males or species with multimale groups; Moller and Birkhead, 1993) on the flexibility of AR to social challenges. This does not exclude the possibility that breeding dispersal and group composition still remain secondarily involved parameters by being related with male-male aggressiveness and mating strategy, which were shown to modulate the androgen response rates. Alternatively, this may add another example that explanations at an intraspecific level differ from those at the interspecific level.

In conclusion, our study pinpointed the importance of controlling for phylogenetic effects while examining relationships among phenotypic traits across species (Felsenstein, 1985; Harvey and Pagel, 1991; Martins and Hansen, 1997). Comparative studies allow to extend insights from studies at the population level and to generate predictions at the interspecific level (MacDougall-Shackleton and Ball, 1999; Dunn et al., 2001). The knowledge that changes of mating strategy and of territorial male-male aggressiveness rather than altered degrees of paternal care in general were related to the evolution of different androgen response rates in male birds, may contribute to the discussion of the applicability of the predictions of the challenge hypothesis throughout the vertebrates (vertebrates in general: Hirschen- 
hauser and Oliveira, 2000; lizards: Thompson and Moore, 1992; but see Houck and Woodley, 1995; teleosts: Cardwell and Liley, 1991; Pankhurst and Barnett, 1993; Oliveira et al., 1996, 2001c, 2002; mammals: Cavigelli and Pereira, 2000; Nunes et al., 2000; but see Creel et al. 1993). Only the change to extremely high paternal investment (i.e., paternal incubation among the altricial passerines) contributed to the evolution of different androgen response rates. Finally, this study demonstrated that mating strategy and male-male aggressiveness are the primary functions of androgen-dependent male behavior, whereas paternal investment is not a function of but rather constrained by high androgen levels.

\section{Acknowledgments}

We thank A.V.M. Canario, L.A. Carneiro, M. Hau, J. Nesbitt, P. Sá Pessoa, A. Peters, A.F.H. Ros, P.J. Sharp, and C. Vleck for helping to find primary sources; G. Ball, K. Kotrschal, A.F.H. Ros, B. Taborsky, M. Taborsky, J.C. Wingfield and three anonymous referees for stimulating discussions on this topic; D. Podolsky (KLIVV, Vienna), the Konrad Lorenz Forschungsstelle (Grünau, Austria), and the staff of the British Library (section "Science and Technology") for helping with access to library sources. This study was funded by a research grant from the Fundação para a Ciência e a Tecnologia (FCT; PRAXIS XXI/P/BIA/ 10251/98). KH was being supported by a post-doctoral grant from FCT; PRAXIS XXI/BPD/20142/99).

\section{References}

Akesson, T.R., Raveling, D.G., 1981. Endocrine and body weight changes of nesting and non-nesting Canada geese. Biol. Reprod. 25, 792-804.

Alatalo, R.V., Höglund, J., Lundberg, A., Rintamäki, P.T., Silverin, B., 1996. Testosterone and male mating success on the black grouse leks. Proc. R. Soc. Lond. B 263, 1697-1702.

Ball, G.F., Wingfield, J.C., 1987. Changes in plasma luteinizing hormone and sex steroid hormones in relation to multiple-broodedness and nest-site density in male starlings. Physiol. Zool. 60, 191-199.

Beletsky, L.D., Orians, G.H., Wingfield, J.C., 1989. Relationships of steroid hormones and polygyny to territorial status, breeding experience, and reproductive success in male red-winged blackbirds. Auk 106, 107-117.

Beletsky, L.D., Orians, G.H., Wingfield, J.C., 1992. Year-to-year patterns of circulating levels of testosterone and corticosterone in relation to breeding density, experience, and reproductive success of the polygynous red-winged blackbird. Horm. Behav. 26, 420-432.

Belthoff, J.R., Dufty Jr., A.M., 1994. Plumage variation, plasma steroids and social dominance in male house finches. Condor 96, 614-625.

Berry, H.H., Millar, R.P., Louw, G.N., 1979. Environmental cues influencing the breeding biology and circulating levels of various hormones and triglycerides in the cape cormorant. Comp. Biochem. Physiol. 62 (A), 879-884.

Borgia, G., Wingfield, J.C., 1991. Hormonal correlates of bower decoration and sexual display in the satin bowerbird (Ptilorhynchus violaceus). Condor 93, 935-942.

Bottoni, L., Massa, R., Lea, R.W., Sharp, P.J., 1993. Mate choice and reproductive success in red-legged partridge (Alectoris rufa). Horm. Behav. 27, 308-317.
Burger, A.E., Millar, R.P., 1980. Seasonal changes of sexual and territorial behaviour and plasma testosterone levels in male lesser sheatbills (Chionis minor). Zeitschr. Tierpsychol. 52, 397-406.

Cardwell, J.R., Liley, N.R., 1991. Androgen control of social status in males of a wild population of stoplight parrot fish, Sparisoma viride (Scaridae). Horm. Behav. 25, 1-18.

Cavigelli, S., Pereira, M.E., 2000. Mating aggression and fecal testosterone levels in male ring-tailed lemurs (Lemur catta). Horm. Behav. 37, $246-255$.

Chandler, C.R., Ketterson, E.D., Nolan Jr., V., 1997. Effects of testosterone on use of space by male dark-eyed juncos when their mates are fertile. Anim. Behav. 54, 543-549.

Clutton-Brock, T.H., 1991. The Evolution of Parental Care. Princeton University Press, Princeton, NJ.

Cockrem, J.F., Potter, M.A., 1990. Reproductive endocrinology of the North Island brown kiwi Apteryx australis mantelli, in: Bell, B.D., Cossee, R.O., Flux, J.E.C., Heather, B.D., Hitchmough, R.A., Robertson, C.J.R., Williams, M.J. (Eds.), Acta XX Congressus Internationalis Ornithologici, New Zealand Ornithological Congress Trust Board, Wellington, NZ, pp. 2093-2101.

Cockrem, J.F., Seddon, P.J., 1994. Annual cycle of sex steroids in the yellow-eyed penguin (Megadyptes antipodes) on South Island, New Zealand. Gen. Comp. Endocrinol. 94, 113-121.

Cramp, S., 1998. The Complete Birds of the Western Palaearctic. University Press, OptiMedia, CD-ROM, Oxford.

Creel, S., Wildt, D.E., Monfort, S.L., 1993. Aggression, reproduction, and androgens in wild dwarf mongooses: a test of the challenge hypothesis. Am. Nat. 141, 816-825.

Degen, A.A., Weil, S., Rosenstrauch, A., Kam, M., Dawson, A., 1994. Seasonal plasma levels of LH and steroid hormones in male and female domestic ostriches (Struthio camelus). Gen. Comp. Endocrinol. 93, 21-27.

De Ridder, E., Pinxten, R., Eens, M., 2000. Experimental evidence of a testosterone-induced shift from paternal to mating behaviour in a facultatively polygynous songbird. Behav. Ecol. Sociobiol. 49, 24-30.

Dittami, J.P., 1981. Seasonal changes in the behavior and plasma titers of various hormones in barheaded geese, Anser indicus. Zeitschr. Tierpsychol. 55, 289-324.

Dittami, J.P., Gwinner, E., 1990. Endocrine correlates of seasonal reproduction and territorial behavior in some tropical passerines, in: Wada, M., Ishii, S., Scanes, C.G. (Eds.), Endocrinology of Birds: Molecular to Behavioral, Japan Sci. Soc. Press/Springer-Verlag, Tokyo, Berlin, pp. 225-233.

Dittami, J.P., H., Hoi, Sageder, G., 1991. Parental investment and territo$\mathrm{rial} / \mathrm{sexual}$ behavior in male and female reed warblers: are they mutually exclusive? Ethology 88, 249-255.

Dobson, A.J., 1990. An Introduction to Generalized Linear Models. Chapman and Hall, London.

Dufty Jr., A.M., 1989. Testosterone and survival: a cost of aggressiveness? Horm. Behav. 23, 185-193.

Dufty, Jr. A.M., 1993. Testosterone concentrations in males of an atypical species: the brood-parasitic brown-headed cowbird, in: Sharp, P., Avian Endocrinology, Journal of Endocrinology, Bristol, pp. 61-72.

Dunn, P.O., Whittingham, L.A., Pitcher, T.E., 2001. Mating systems, sperm competition, and the evolution of sexual dimorphism in birds. Evolution 55, 161-175.

Efron, B., Tibshirani, R.J., 1993. An Introduction to the Bootstrap. Chapman and Hall, New York.

Ellsworth, D.L., Honeycutt, R.L., Silvy, N.J., 1996. Systematics of grouse and ptarmigan determined by nucleotide sequences of the mitochondrial cytochrome- $b$ gene. Auk 113, 811-822.

Felsenstein, J., 1985. Phylogenies and the comparative method. Am. Nat. $125,1-15$.

Fivizzani, A.J., Oring, L.W., 1986. Plasma steroid hormones in relation to behavioral sex role reversal in the spotted sandpiper, Actitis macularia. Biol. Reprod. 35, 1195-1201. 
Fivizzani, A.J., Colwell, M.A., Oring, L.W., 1986. Plasma steroid hormone levels in free-living Wilson's phalaropes, Phalaropus tricolor. Gen. Comp. Endocrinol. 62, 137-144.

Fornasari, L., Bottoni, L., Schwabl, H., Massa, R., 1992. Testosterone in the breeding cycle of the red-backed shrike Lanius collurio. Ethol. Ecol. Evol. 4, 193-196.

Fraissinet, M., Varriale, B., Pierantoni, R., Caliendo, M. F., Di Matteo, L., Bottoni, L., Milone, M., 1987. Annual testicular activity in the gray partridge (Perdix perdix). Gen. Comp. Endocrinol. 68, 28-32.

Garland, T., Midford, P.E., Ives, A.R., 1999. An introduction to phylogenetically based statistical methods, with a new method for confidence intervals on ancestral states. Am. Zool. 39, 374-388.

Glutz von Blotzheim, U.N., Bauer, K.M., Bezzel, E., 1981. Handbuch der Vögel Mitteleuropas, Vol. 5. Galliformes and Gruiformes, Akadem, Verlagsgesellschaft, Wiesbaden.

Gorman, M.L., 1977. Sexual behaviour and plasma androgen concentrations in the male Eider duck (Somateria mollissima). J. Reprod. Fertil. $49,225-230$.

Gratto-Trevor, C.L., Fivizzani, A.J., Oring, L.W., Cooke, F., 1990. Seasonal changes in gonadal steroids of a monogamous versus a polyandrous shorebird. Gen. Comp. Endocrinol. 80, 407-418.

Groscolas, R., Jallageas, M., Goldsmith, A., Assenmacher, I., 1986. The endocrine control of reproduction and molt in male and female emperor (Aptenodytes forsteri) and adelie (Pygoscelis adeliae) penguins. Gen. Comp. Endocrinol. 62, 43-53.

Gwinner, E., Roedl, T., Schwabl, H., 1994. Pair territoriality of wintering stonechats: behaviour, function and hormones. Behav. Ecol. Sociobiol. 34, 321-327.

Hannon, S.J., Wingfield, J.C., 1990. Endocrine correlates of territoriality, breeding stage, and body molt in free-living willow ptarmigan of both sexes. Can. J. Zool. 68, 2130-2134.

Harvey, P.H., Pagel, M.D., 1991. The Comparative Method in Evolutionary Biology. Oxford University Press, Oxford.

Hector, J.A.L., Follett, B.K., Prince, P.A., 1986a. Reproductive endocrinology of the black-browed albatross Diomedea melanophris and the grey-headed albatross D. chrysostoma. J. Zool. 208, 237-253.

Hector, J.A.L., Croxall, J.P., Follett, B.K., 1986b. Reproductive endocrinology of the wandering albatross Diomedea exulans in relation to biennial breeding and deferred sexuality. Ibis $128,9-22$.

Hegner, R.E., Wingfield, J.C., 1986. Behavioral and endocrine correlates of multiple brooding in the semicolonial house sparrow Passer domesticus. I. Males. Horm. Behav. 20, 294-326.

Hegner, R.E., Wingfield, J.C., 1987. Effects of experimental manipulation of testosterone levels on parental investment and breeding success in male house sparrows. Auk 104, 462-469.

Helbig, A.J., Seibold, I., 1999. Molecular phylogeny of Palearctic-African Acrocephalus and Hippolais warblers (Aves: Sylviidae). Mol. Phyl. Evol. 11, 246-260.

Hirschenhauser, K., Oliveira, R.F., 2000. The 'challenge hypothesis' revisited: a meta-analysis. Trabajos Inst. Cajal 77, 305.

Hirschenhauser, K., Möstl, E., Kotrschal, K., 1999. Seasonal patterns of sex steroids determined from feces of different social categories of greylag geese Anser anser. Gen. Comp. Endocrinol. 114, 67-79.

Hirschenhauser, K., Möstl, E., Wallner, B., Dittami, J., Kotrschal, K., 2000. Endocrine and behavioural responses of male Greylag geese (Anser anser) to pairbond challenges during the reproductive season Ethology 106, 63-78.

Houck, L.D., Woodley, S.K., 1995. Field studies of steroid hormones and male reproductive behavior in amphibians, in: Heatwole, H., Sullivan, B.K. (Eds.), Amphibian Biology, Vol. 2: Social Behavior, Surrey Beatty and Sons, New South Wales, Australia, pp. 677-703.

Hoyo, J. del, Elliott, A., Sargatel, J., 1992. Handbook of the Birds of the World Vol. 1: Ostrich to Ducks, Lynx Edicions, Barcelona.

Hoyo, J. del, Elliott, A., Sargatel, J., 1996. Handbook of the Birds of the World, Vol. 3: Hoatzin to Auks, Lynx Edicions, Barcelona.

Hunt, K., Wingfield, J.C., Astheimer, L.B., Buttemer, W.A., Hahn, T.P., 1995. Temporal patterns of territorial behavior and circulating testos- terone in the Lapland Longspur and other Arctic passerines. Am. Zool. $35,274-284$.

Hunt, K.E., Hahn, T.P., Wingfield, J.C., 1999. Endocrine influences on parental care during a short breeding season: testosterone and male parental care in Lapland longspurs (Calcarius lapponicus). Behav. Ecol. Sociobiol. 45, 360-369.

Ishii, S., Wada, M., Wakabayashi, S., Sakai, H., Kubodera, Y., Yamaguchi, N., Kikuchi, M., 1994. Endocrinological studies for artificial breeding of the Japanese ibis, Nipponia nippon, an endangered avian species in Asia. J. Biosci. 19, 491-502.

Johnsgard, P.A., 1994. Arena birds. Sexual Selection and Behavior. Smithsonian Institution Press, Washington, London.

Johnson, K.P., Lanyon, S.M., 1999. Molecular systematics of the grackles and allies, and the effect of additional sequence (cyt $b$ and ND2). Auk $116,759-76$

Ketterson, E.D., Nolan Jr., V., 1992. Hormones and life histories: an integrative approach. Am. Nat. 140, S33-S62.

Khan, M.Z., McNabb, F.M.A., Walters, J.R., Sharp, P.J., 2001. Patterns of testosterone and prolactin concentrations and reproductive behavior of helpers and breeders in cooperatively breeding red-cockaded woodpecker (Picoides borealis). Horm. Behav. 40, 1-13.

Kimball, R.T., Zwartjes, P.W., Crowe, T.M., Ligon, J.D., 1999. A molecular phylogeny of the pheasants and partridges suggests that these lineages are not monophyletic. Mol. Phyl. Evol. 11, 38-54.

Klicka, J., Johnson, K.P., Lanyon, S.M., 2000. New World nine-primaried oscine relationships: constructing a mitochondrial DNA framework. Auk 117, 321-336.

Kofuji, H., Kanda, M., Oishi, T., 1993. Breeding cycles and fecal gonadal steroids in the brown dipper Cinclus pallasii. Gen. Comp. Endocrinol. 91, 216-223.

Kotrschal, K., Dittami, J., Hirschenhauser, K., Möstl, E., Péczely, P., 2000. Effects of physiological and social challenges in different seasons on fecal testosterone and corticosterone in male domestic geese (Anser domesticus). Acta Ethol. 2, 115-122.

Krishnapradasan, T.N., Kotak, V.C., Sharp, P.J., Schmedemann, R., Haase, E., 1988. Environmental and hormonal factors in seasonal breeding in free-living male Indian rose-ringed parakeets (Psittacula krameri). Horm. Behav. 22, 488-496.

Leisler, B., Winkler, H., Wink, M., 2002. Evolution of breeding systems in acrocephaline warblers. Auk 119, 379-390.

Levin, R.N., Wingfiled, J.C., 1992. The hormonal control of territorial aggression in tropical birds. Om. Scand. 23, 284-291.

Lisano, M.E., Kennamer, J.E., 1977. Seasonal variations in plasma testosterone level in male eastern wild turkeys. J. Wildl. Manage. 41, 184188 .

Lormée, H., Jouventin, P., Lacroix, A., Lallemand, J., Chastel, O., 2000. Reproductive endocrinology of tropical seabirds: sex-specific patterns of LH, steroids, and prolactin secretion in relation to parental care. Gen. Comp. Endocrinol. 119, 413-428.

Lynn, S.E., Hayward, L.S., Benowitz-Fredericks, Z.M., Wingfield, J.C., 2002. Behavioural insensitivity to supplementary testosterone during the parental phase in the chestnut-collared longspur, Calcarius ornatus. Anim. Behav. 63, 795-803.

MacDougall-Shackleton, S.A., Ball, G.F., 1999. Comparative studies of sex differences in the song-control system of songbirds. TINS 22, 432-436

Malecki, I.A., Marin, G.B., O’Malley, P.J., Meyer, G.T., Talbot, R.T., Sharp, P.J., 1998. Endocrine and testicular changes in a short-day seasonally breeding bird, the emu (Dromaius novaehollandiae), in SW-Australia. Anim. Reprod. Sci. 53, 143-155.

Marler, C.A., Moore, M.C., 1988. Evolutionary costs of aggression revealed by testosterone manipulations in free-living male lizards. Behav. Ecol. Sociobiol. 23, 21-26.

Martins, E.P., Hansen, T.F., 1997. Phylogenies and the comparative method: a general approach to incorporating phylogenetic information into the analysis of interspecific data. Am. Nat. 149, 646-667. 
Mauget, R., Jouventin, P., Lacroix, A., Ishii, S., 1994. Plasma LH and steroid hormones in king penguin (Aptenodytes patagonicus) during the onset of the breeding cycle. Gen. Comp. Endocrinol. 93, 36-43.

Mays, N.A., Vleck, C.M., Dawson, J., 1991. Plasma LH, steroid hormones, behavioral role, and nest stage in cooperatively breeding Harris' hawks (Parabuteo unicinctus). Auk 108, 619-637.

McDonald, P.G., Buttemer, W.A., Astheimer, L.B., 2001. The influence of testosterone on territorial defence and parental behavior in male freeliving rufous whistlers, Pachycephala rufiventris. Horm. Behav. 39, 185-194.

Meijer, T., Schwabl, H., 1989. Hormonal patterns in breeding and nonbreeding kestrels, Falco tinnunculus: field and laboratory studies. Gen. Comp. Endocrinol. 74, 148-160.

Møller, A.P., Birkhead, T.R., 1993. Cuckoldry and sociality: a comparative study of birds. Am. Nat. 142, 118-140.

Møller, A.P., Cuervo, J.J., 2000. The evolution of paternity and paternal care in birds. Behav. Ecol. 11, 472-485.

Moore, M.C., 1982. Hormonal response of free-living male white-crowned sparrows to experimental manipulation of female sexual behavior. Horm. Behav. 16, 323-329.

Moore, M.C., 1991. Applications of organization-activation theory to alternative male reproductive strategies: a review. Horm. Behav. 25, $154-179$.

Morse Nice, M., 1943. Studies in the Life History of the Song Sparrow: The Behavior of the Song Sparrow and Other Passerines, Vol. III. Dover Publ., New York.

Narasimhacharya, A.V.R.L., Kotak, V.C., Sharp, P.J., 1988. Environmental and hormonal interactions in the regulation of seasonal breeding in free-living male Indian baya weaver birds (Ploceus philippinus). J. Zool. 215, 239-248.

Nunes, S., Fite, J.E., French, J.A., 2000. Variation in steroid hormones associated with infant care behaviour and experience in male marmosets (Callithrix kuhlii). Anim. Behav. 60, 857-865.

O'Connell, M.E., Reboulleau, C., Feder, H.H., Silver, R., 1981. Social interactions and androgen levels in birds. Gen. Comp. Endocrinol. 44, $454-463$

Oliveira, R.F., Almada, V.C., Canário, A.V.M., 1996. Social modulation of sex steroid concentrations in the urine of male cichlid fish Oreochromis mossambicus. Horm. Behav. 30, 2-12.

Oliveira, R.F., Ros, A.F.H., Hirschenhauser, K., Canario, A.V.M., 2001a Androgens and mating systems in fish: intra- and interspecific analyses, in: Goos, H.J.T., Rastogi, R.K., Vaudry, H., Pierantoni, R. (Eds.), Perspectives in Comparative Endocrinology: Unity and Diversity, Monduzzi Editore, Sorrento, Italy, 985-993.

Oliveira, R.F., Canario, A.V.M., Grober, M.S., Serrão Santos, R., 2001b. Endocrine correlates of male polymorphism and alternative reproductive tactics in the Azorean rock-pool blenny, Parablennius sanguinolentus parvicornis. Gen. Comp. Endocrinol. 121, 278-288.

Oliveira, R.F., Lopes, M., Carneiro, L.A., Canario, A.V.M., 2001c. Watching fights raises fish hormone levels. Nature 409, 475.

Oliveira, R.F., Hirschenhauser, K., Carneiro, L.A., Canario, A.V.M., 2002 Social modulation of androgens in male teleost fish. Comp. Biochem. Physiol B 132, 203-215.

Overall, J.E., Spiegel, D.K., 1969. Concerning least squares analysis of experimental data. Psychol. Bull. 72, 311-322.

Pagel, M.D., Harvey, P.H., 1988. Recent developments in the analysis of comparative data. Qu. Rev. Biol. 63, 413-440.

Pankhurst, N.W., Barnett, P.W., 1993. Relationship of population density, territorial interaction and plasma levels of gonadal steroids in spawning male demoiselles Chromis dispilus (Pisces: Pomacentridae). Gen. Comp. Endocrinol. 90, 168-176.

Patten, M.A., Fugate, M., 1998. Systematic relationships among the emberizid sparrows. Auk 115, 412-424.

Paulke, E., Haase, E., 1978. A comparison of seasonal changes in the concentrations of androgens in the peripheral blood of wild and domestic ducks. Gen. Comp. Endocrinol. 34, 381-390.
Péczely, P., Pethes, G., 1979. Alterations in plasma sexual steroid concentrations in the collared dove (Streptopelia decaocto) during the sexual maturation and reproduction cycle. Acta Physiol. Acad. Sci. Hung. 54, 161-170.

Péczely, P., Pethes, G., 1982. Seasonal cycle of gonadal, thyroid, and adrenocortical function in the rook (Corvus frugilegus). Acta Physiol. Acad. Sci. Hung. 59, 59-73.

Peters, A., 2000. Testosterone treatment is immunosuppressive in superb fairy-wrens, yet free-living males with high testosterone are more immunocompetent. Proc. R. Soc. Lond. B 267, 883-889.

Price, T., 1997. Correlated evolution and independent contrasts. Philos. Trans. R. Soc. Lond. B 352, 519-529.

Ptacek, M.B., Travis, J., 1998. Hierarchical patterns of covariance between morphological and behavioural traits. Anim. Behav. 56, 1044-1048.

Reyer, H.U., Dittami, J.P., Hall, M.R., 1986. Avian helpers at the nest: are they psychologically castrated? Ethology 71, 216-228.

Rissman, E., Wingfield, J.C., 1984. Hormonal correlates of polyandry in the spotted sandpiper, Actitis macularia. Gen. Comp. Endocrinol. 56, 401-405.

Rohlf, F.J., 2001. Comparative methods for the analysis of continuous variables: geometric interpretations. Evolution 55, 2143-2160.

Romero, L.M., Soma, K.K., O'Reilly, K.M., Suydam, R., Wingfield, J.C., 1998. Hormones and territorial behavior during breeding in snow buntings (Plectrophenax nivalis): an arctic-breeding songbird. Horm. Behav. 33, 40-47.

Ros, A.F.H., Groothuis, T.G.G., Apanius, V., 1997. The relation among gonadal steroids, immunocompetence, body mass, and behavior in young black-headed gulls (Larus ridibundus). Am. Nat. 150, 201-219.

Saint Jalme, M., Williams, J., Mickaelian, I., Paillat, P., 1996. Seasonal variation of LH, sex steroids, body mass, molt, display, and laying in two subspecies of Houbara bustard, Chlamydotis undulata macqueenii and $C$. $u$. undulata, housed in outdoor cages under natural conditions. Gen. Comp. Endocrinol. 102, 102-112.

Sakai, H., Ishii, S., 1986. Annual cycles of plasma gonadotropins and sex steroids in Japanese common pheasants, Phasianus colchicus versicolor. Gen. Comp. Endocrinol. 63, 275-283.

Schoech, S.J., Mumme, R.L., Moore, M.C., 1991. Reproductive endocrinology and mechanisms of breeding inhibition in cooperatively breeding Florida scrub jays (Aphelocoma c. coerulescens). Condor 93, 354364.

Schwabl, H., Wingfield, J.C., Farner, D.S., 1980. Seasonal variation in plasma levels of luteinizing hormone and steroid hormones in the European blackbird Turdus merula. Vogelwarte 30, 283-294.

Searcy, W.A., Yasukawa, K., Lanyon, S., 1999. Evolution of polygyny in the ancestors of red-winged blackbirds. Auk 116, 5-19.

Seiler, H.W., Gahr, M., Goldsmith, A.R., Güttinger, H.-R., 1992. Prolactin and gonadal steroids during the reproductive cycle of the Bengalese finch (Lonchura striata var. domestica, Estrilidae), a nonseasonal breeder with biparental care. Gen. Comp. Endocrinol. 88, 83-90.

Sibley, C.G., Ahlquist, J.E., 1990. Phylogeny and Classification of Birds. A Study in Molecular Evolution. Yale University Press, New Haven, London.

Silverin, B., 1980. Effects of long-acting testosterone treatment on freeliving pied flycatchers, Ficedula hypoleuca, during the breeding period. Anim. Behav. 28, 906-912.

Silverin, B., 1990. Some aspects of the seasonal changes in circulating levels of LH and testosterone in willow tits Parus montanus and great tits Parus major, in: Bell, B.D., Cossee, R.O., Flux, J.E.C., Heather, B.D., Hitchmough, R.A., Robertson, C.J.R., Williams, M.J. (Eds.), Acta XX Congressus Internationalis Ornithologici, Christchurch, New Zealand, pp. 2081-2091.

Silverin, B., Wingfield, J.C., 1982. Patterns of breeding behaviour and plasma levels of hormones in a free-living population of pied flycatchers, Ficedula hypoleuca. J. Zool. 198, 117-129.

Stacey, P.B., Koenig, W.D., 1990. Cooperative Breeding in Birds. Longterm Studies of Ecology and Behavior. Cambridge Univ. Press, Cambridge, New York. 
Stearns, S., 1989. Trade-offs in life-history evolution. Funct. Ecol. 3, $259-268$

Stokkan, K.-A., Sharp, P.J., 1990. Seasonal breeding in high-arctic ptarmigan, in: Wada, M., Ishii, S., Scanes, C.G. (Eds.), Endocrinology of Birds: Molecular to Behavioral, Japan Sci. Soc. Press/Springer-Verlag, Tokyo, Berlin, pp. 251-258.

Tabachnick, B.G., Fidell, L.S., 2001. Using Multivariate Statistics. Allyn and Bacon, Boston.

Thompson, C.W., Moore, M.C., 1992. Behavioral and hormonal correlates of alternative reproductive strategies in a polygynous lizard: tests of the relative plasticity and challenge hypothesis. Horm. Behav. 26, 568585.

Van Duyse, E., Pinxten, R., Eens, M., 2000. Does testosterone affect the trade-off between investment in sexual/territorial behaviour and parental care in male great tits? Behaviour 137, 1503-1515.

Van Duyse, E., Pinxten, R., Eens, M., 2002. Effects of testosterone on song, aggression, and nestling feeding behavior in male great tits. Parus major. Horm. Behav. 41, 178-186.

Vleck, C.M., 1993. Hormones, reproduction, and behaviour in birds of the Sonoran desert, in: Sharp, P.J. (Ed.), Avian Endocrinology, Journal of Endocrinology, Bristol, pp. 73-86.

Vleck, C.M., Brown, J.L., 1999. Testosterone and social and reproductive behaviour in Aphelocoma jays. Anim. Behav. 58, 943-951.

Vleck, C.M., Dobrott, S.J., 1993. Testosterone, antiandrogen, and alloparental behavior in bobwhite quail foster fathers. Horm. Behav. 27, 92-107.

Wada, M., Shimizu, T., Kobayashi, S., Yatani, A., Sandaiji, Y., Ishikawa, T., Takemure, E., 1999. Behavioral and hormonal basis of polygynous breeding in male bush warblers (Cettia diphone). Gen. Comp. Endocrinol. 116, 422-432.

Welty, J.C., 1982. The Life of Birds. Saunders College Publishing, Philadelphia, New York.

Westneat, D.F., Sherman, P.W., 1997. Density and extra-pair fertilizations in birds: a comparative analysis. Behav. Ecol. Sociobiol. 41, 205-215.

Williams, T.D., 1992. Reproductive endocrinology of Macaroni (Eudyptes chrysolophus) and Gentoo (Pygoscelis papua) penguins. Gen. Comp. Endocrinol. 85, 230-240.
Willis, E.O., 1972. The behavior of spotted antbirds. Ornithol. Monogr. 10, $1-157$.

Wikelski, M., Hau, M., Wingfield, J.C., 1999. Social instability increases plasma testosterone in a year-round territorial neotropical bird. Proc. R. Soc. Lond. B 266, 551-556.

Wingfield, J.C., 1984a. Environmental and endocrine control of reproduction in the song sparrow, Melospiza melodia. II: Agonistic interactions as environmental information stimulating secretion of testosterone. Gen. Comp. Endocrinol. 56, 417-424.

Wingfield, J.C., 1984b. Androgens and mating systems: testosterone induced polygyny in a normally monogamous bird. Auk 4, 665-671.

Wingfield, J.C., 1990. Interrelationships of androgens, aggression, and mating systems, in: Wada, M.S., Ishii, S., Scanes, C.G. (Eds.), Endocrinology of Birds: Molecular to Behavioral, Japan Sci. Soc. Press, Springer-Verlag, Tokyo, Berlin, pp. 187-205.

Wingfield, J.C., 1994. Control of territorial aggression in a changing environment. Psychoneuroendocrinology 19, 709-721.

Wingfield, J.C., Hegner, R-.E., Dufty Jr., A.M., Ball, G. F., 1990. The 'challenge hypothesis': theoretical implications for patterns of testosterone secretion, mating systems and breeding strategies. Am. Nat. 136, $829-846$.

Wingfield, J.C., Doak, D., Hahn, T.P., 1993. Integration of environmental cues regulating transitions of physiological state, morphology and behavior, in: Sharp, P.J. (Ed.), Avian Endocrinology, Journal of Endocrinology, Bristol, UK, pp. 111-122.

Wingfield, J.C., Jacobs, J., Hillgarth, N., 1999a. Ecological constraints and the evolution of hormone-behavior interrelationships. Am. Nat. 136, $829-846$.

Wingfield, J.C., Ramos-Fernandez, G., Nunez-de la Mora, A., Drummond, II., 1999b. The effects of an 'El Nino' southern oscillation event on reproduction in male and female blue-footed boobies. Sula nebouxii. Gen. Comp. Endocrinol. 114, 163-172.

Wingfield, J.C., Jacobs, J.D., Tramontin, A.D., Perfito, N., Meddle, S., Maney, D.L., Soma, K., 2000. Toward an ecological basis of hormonebehavior interactions in reproduction of birds, in: Wallen, K., Schneider, J.E. (Eds.), Reproduction in Context. Social and Environmental Influences on Reproduction, A Bradford Book, MIT Press, Cambridge, MA, London, pp. 85-128. 